\title{
One Way Fluid Solid Coupling Analysis of Gas Mask Based on ANSYS-CFX
}

\author{
Wei Huang ${ }^{1 a^{*}}$, Xiaoli Jin ${ }^{1, b}$ and Liying Wang ${ }^{3, c}$ \\ ${ }^{1}$ No.2 Xuefu Road, Weiyang District, Xi'an City, Shanxi Province, China \\ ${ }^{3}$ West Building, Garden North Road Haidian District, Beijing City, China \\ ahuangwei_9092@163.com, b372847024@qq.com, cadam61@sina.com
}

\section{Keyword: Gas-mask; Workbench; ANSYS-CFX; Fluid solid coupling}

\begin{abstract}
Gas mask in the process of wearing, in the role of breathing air flow, so that the uneven distribution of pressure inside the cover body, resulting in deformation of the cover body structure, so that the protective performance of the gas mask failure. In this paper, this paper takes the gas mask as an example, through the UG 3D graphics software, get gas mask geometric model. In the human body respiration process, the maximum inspiratory time, using ANSYS analysis software, on the Workbench platform, Through the analysis of software CFX in direct access to cover the airflow field in the pressure from the fluid calculation of fluid solid coupling, and then transfer the fluid pressure load to Static Structural static analysis module, transmission by the data, the mask deformation is more reasonable and more accurate numerical simulation. Through the analysis of the gas mask cover body stress position and the size of the stress and the total deformation and size of the gas mask cover.
\end{abstract}

\section{Introduction}

The mask body is connected with each part of the mask body frame, is an important part of gas masks, which greatly affects the protective performance of gas masks, wearing comfort and service life [1].One of the requirements is to be flexible and to reduce the deformation of the material in order to ensure the comfort and reduce the deformation of the cover structure. In the actual human body wearing a mask, in the suction process due to the uneven distribution of the airflow in the cover body, forming pressure of different sizes, the airflow will exert a force on the cover of the cover body structure deformation, cause serious loss of gas masks and protective properties. For this kind of problem in the past solution, usually just use the gas mask cover body structure analysis. In practical application, this method is unable to calculate the pressure distribution of gas flow in the cover body and in a gas mask cover in different parts of the actual pressure. According to the actual situation, the innovation of the one-way FSI method is first calculated according to the actual pressure in different parts of the cover body, and then through the Workbench platform, the pressure data transmission to the structure analysis module, according to the actual size of the force calculation of cover body deformation parts and deformation, avoid cover structure stress not accurate in the traditional calculation methods of.

In this paper, the state of the maximum inspiratory capacity of a gas mask during inspiration is an example, Workbench Ansys is used to realize the single fluid solid coupling analysis of gas flow and hood structure in gas mask.

\section{Establish Geometric Model}

According to the size information of this gas mask, the structure model of gas mask body was drawn by UG software, Fig 1. 

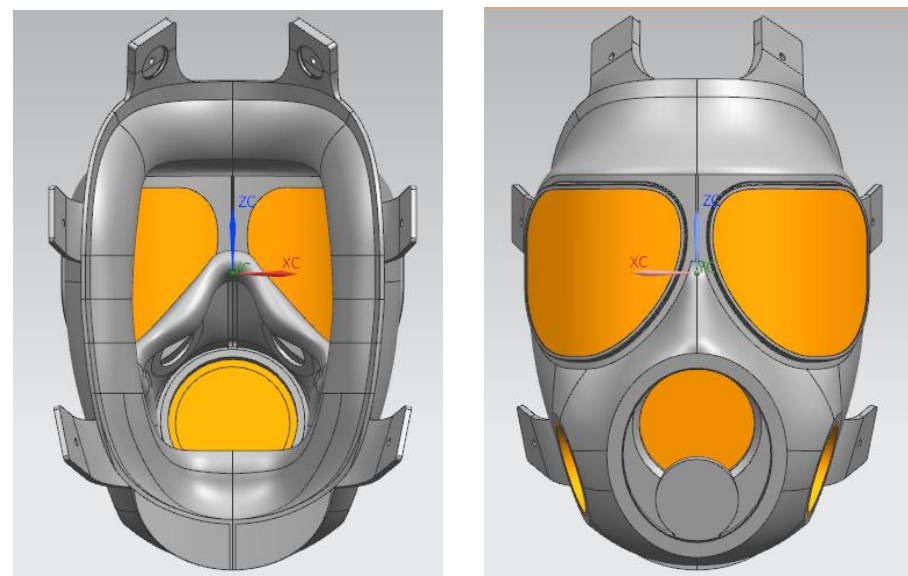

Figure 1. Gas mask body model

In fluid solid coupling analysis, the effect of fluid in gas mask on the structure of the cover body is analyzed,Therefore, in view of the actual situation of this study, we also need to get the human body to wear the face and the gas mask body contact part and the fluid field between the mask,According to the request, this part is composed of the gas space to form a fluid domain,Fig 2.
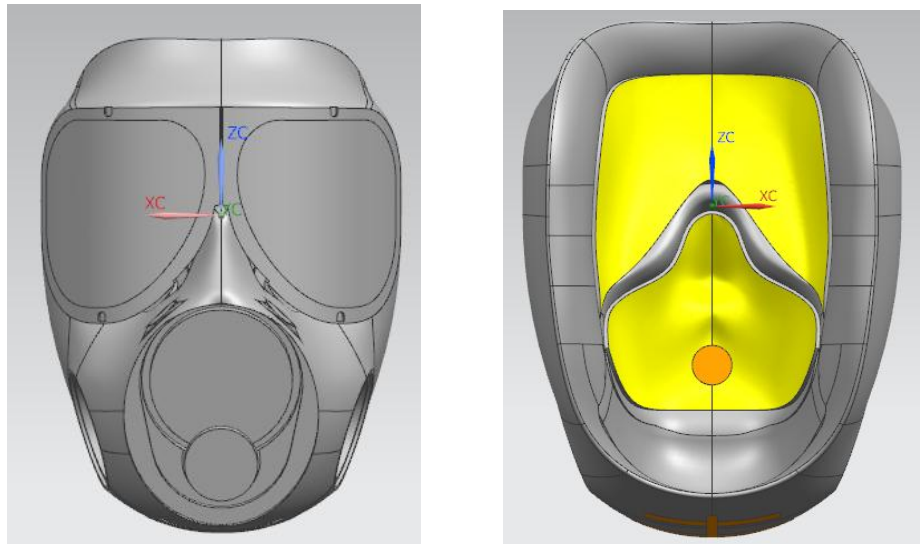

Figure 2. Gas mask fluid field

\section{Dividing Mesh}

Using ICEM CFD ANSYS fluid professional division of the body fluid domain mesh division.The grid is divided into structured and unstructured grid two categories, mainly for hexahedral structured grid, unstructured grid for tetrahedron[2].Considering the fluid domain structure is very complex, this paper adopts a hybrid tetrahedron plus three prism grid division, which uses strong adaptive tetrahedral meshes in fluid domain, at the same time in order to ensure the precision and convergence of the new layers of three prismatic meshes in the boundary layer[3] .In this paper, a tetrahedral mesh is used in the convection body region, the maximum size unit is set to $1 \mathrm{~mm}$, and the expansion layer number is 6,Fig 3.In order to ensure the quality of the grid, the grid quality to take the default less than 0.2 of the mesh to participate in the smooth, CFD ICEM default 5 times.Finally, the total cell number is 4020573 , and the total number of nodes is 1632998 . 

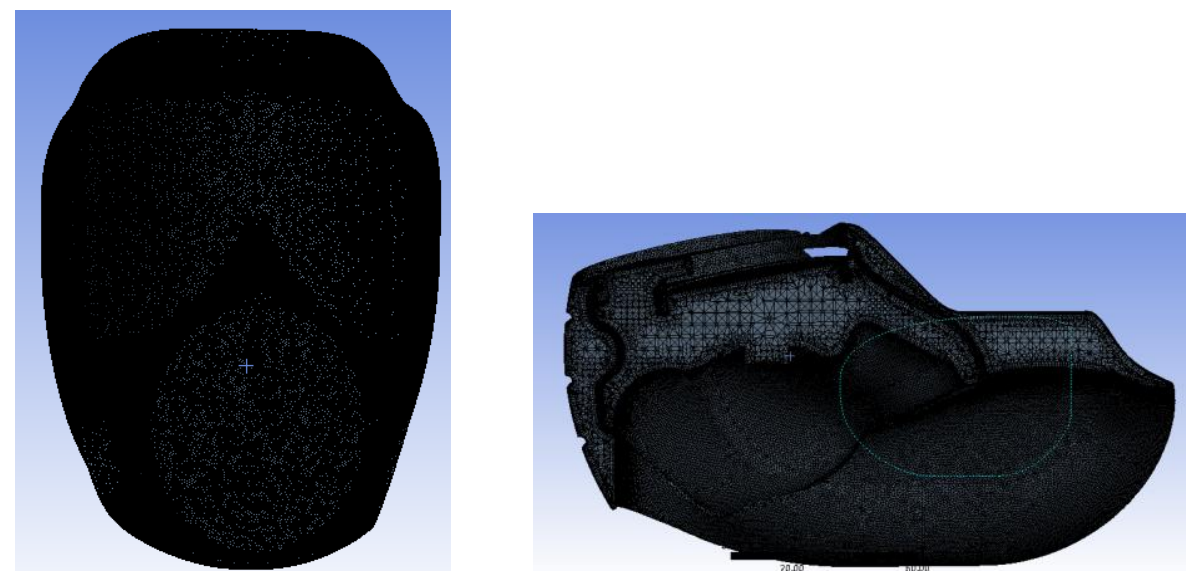

Figure 3. Fluid domain mesh model

\section{One Way Fluid Solid Coupling Analysis of Gas Mask}

4.1 Fluid analysis in CFX. According to the characteristics of air dynamics and fluid mechanics, this paper describes the flow of air in the gas mask in the gas mask in order to form a steady incompressible Newton fluid[4] [5]. And according to the actual situation of the maximum deformation of the cover body, it is used in the maximum inspiratory moment as the simulation object.According to the actual situation of gas mask air inlet $(30 \mathrm{~L} / \mathrm{min})$, the amount of gas in the gas mask is calculated[6].

(1)Analysis type settings

Firstly, according to the simulation method of fluid, the steady state analysis(Steady State).

(2)Select fluid model

CFX was used to simulate the flow field when there are many models can be chosen in this study using standard $\mathrm{k}$ - turbulence model and wall function selection method in boundary layer using Scalable wall function [7] [8].

(3)The setting of flow field boundary conditions

Taking into account the inspiration of the filter canister, the suction valve and the mask of the structure of the formation of resistance, so the suction inlet (IN) of the pressure set $-120 \mathrm{~Pa}$. Set the outlet (OUT) mass flow rate under standard pressure is $0.00065 \mathrm{~kg} / \mathrm{s}$.

After the iteration of the Solver Manager CFX interface shows that the convergence of the residue reached 1e-6, The accuracy of the model is higher, and the convergence is in line with the requirements.Figure 4 shown, The convergence of the $\mathrm{x}, \mathrm{y}, \mathrm{z}$ components and the pressure of the pressure $\mathrm{P}$ are expressed respectively.Fig 5 shown, The convergence of the turbulent kinetic energy $\mathrm{k}$ and the dissipation rate in the air flow model.

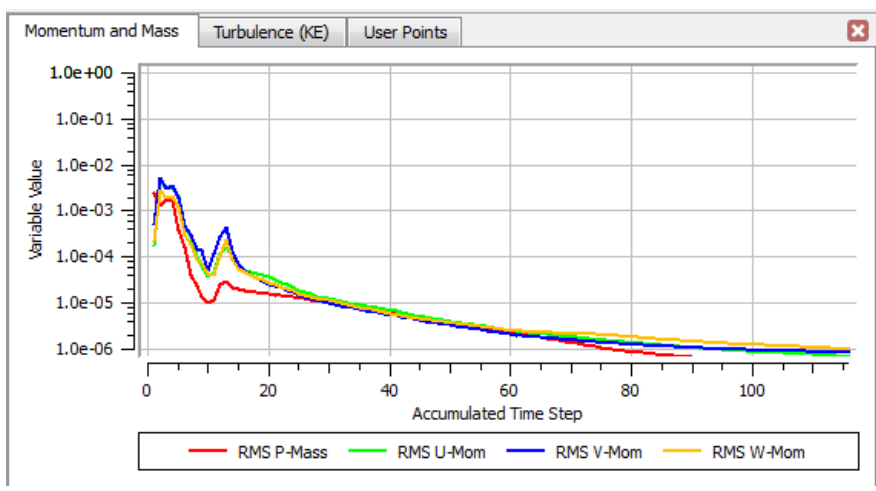

Figure 4. Convergence curves of velocity components and pressure 


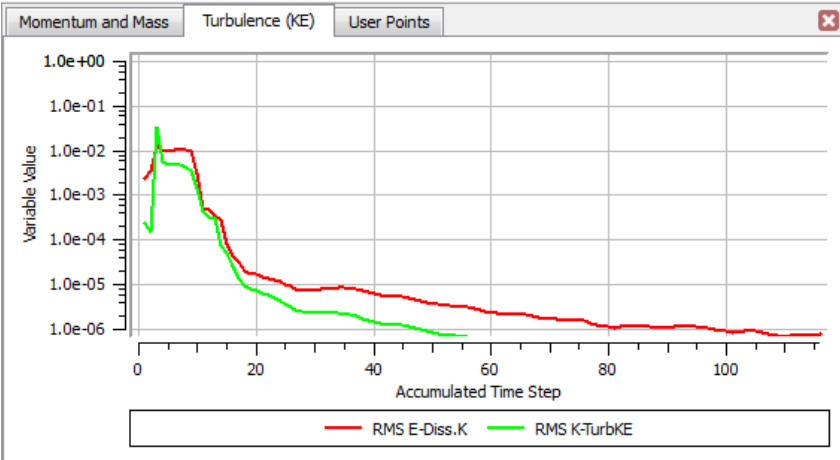

Figure 5. Convergence curves of turbulent kinetic energy and dissipation rate

4.2 Static analysis of enclosure structure. In the static analysis, the basic steps are as follows

(1)Define unit properties

(2)Set material properties, Different elastic modulus, Poisson's ratio and density of the material are arranged according to the different parts of the mask cover body structure [9] [10].Table 1

Table 1 Gas mask body material setting

\begin{tabular}{cccc}
\hline Material Science & ciir & nylon 1010 & polycarbonate \\
\hline Young's modul us(MPa) & 7.84 & 1070 & 2320 \\
Poisson ratio & 0.47 & 0.35 & 0.39 \\
density $\left(\mathrm{kg} / \mathrm{m}^{3}\right)$ & 1050 & 1.05 & 1.9 \\
\hline
\end{tabular}

(3)According to the demand of dividing the proper grid, the grid refinement can make the results more accurate, but will increase the computation time and need more storage space, so you need to weigh the calculation of contradiction between cost and mesh[5]. According to the actual situation, the different parts of the cover can be divided into different quality grids, which can meet the requirements of the solution and reduce the performance requirements of the computer.

(4)Set boundary conditions, Set the fixed load and add the pressure load generated from the CFX analysis.

\section{Results and Analysis}

From the cover body should try to be able to see, gas mask cover body is the largest part of the stress in the front of the cover of the film is about $0.14 \mathrm{MPa}$.Due to the large elastic material, thinner material thickness, the force produced by the inspiratory flow mouth part of a direct role in here, a part of the intake valve through the water blocking cover on the external cover. The body structure of the cover body is a larger part of the cover body rubber parts and material of the non rubber parts of the contact surface, the maximum stress is about $0.05 \mathrm{MPa}$. This is because the inside of the cover body in the air suction generated in the process of force on the cover body, and the cover body material caused by different cover materials with different parts of the deformation, the contact surface of different materials lead to greater stress, Fig6 shown. 

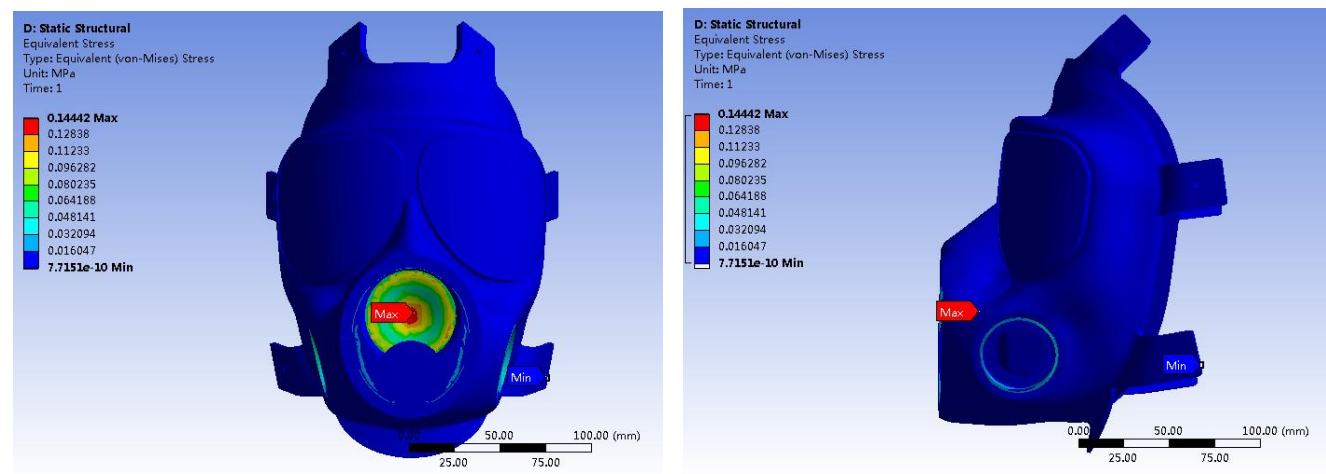

Figure 6. Gas mask cover body

According to the analysis of the stress of the gas mask cover body, it can be seen that the part of the cover body is the most stress. As the material is affected by the greater the stress, the more accelerated the relaxation of the cover body material, resulting in the decline in its stiffness, the more prone to crack phenomenon. Through the fluid solid coupling analysis of the cover body, we can make a more detailed analysis of the site, and then put forward the optimization and improvement of the structure, so that it can get the optimization of the structure.

The total deformation map from the gas mask cover can be seen, gas masks cover comprehensive effects under various loading under the mask on the cover part of the rubber material is easy to deform, the maximum deformation of parts is located on the front of the nose cup film and nose cup call in an air inlet valve and the cover body, the maximum the deformation is about $0.06 \mathrm{~mm}$. The rest is the deformed part and the cover body part and a gas mask cover at the bottom of the rubber material and anti fold wearing claw of the contact, fig 7 shown.
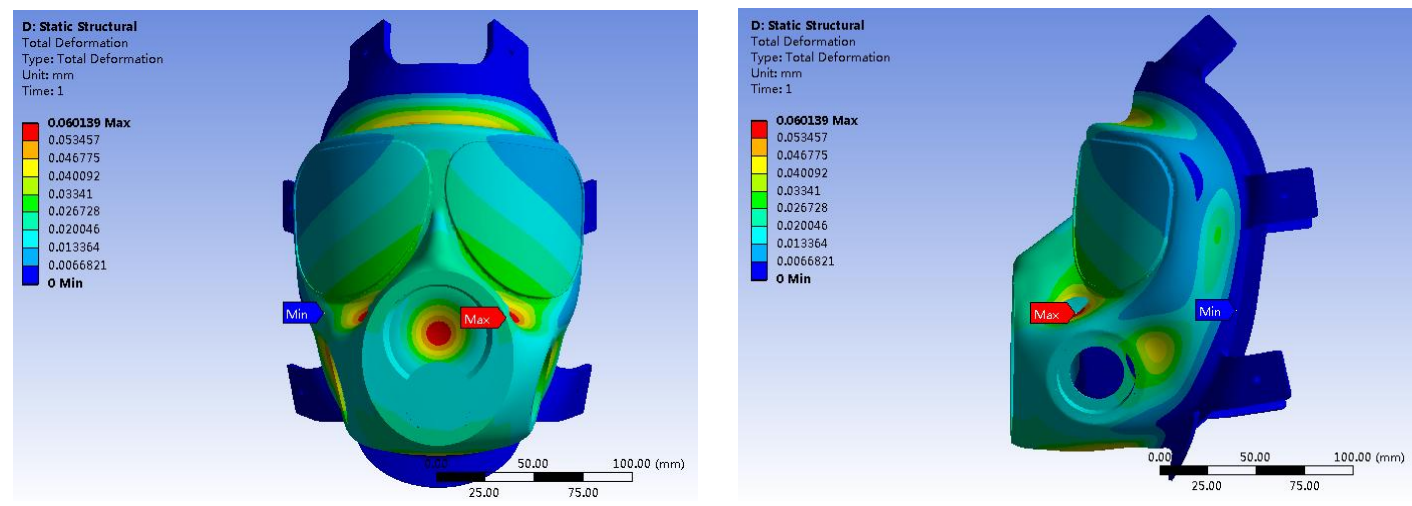

Figure 7. The gas mask cover total deformation

Through the analysis of the gas mask cover deformation, due to fluid shell under the pressure of the cover body structure deformation, with further optimization can reduce the pressure in the suction hood body in the process of making fluid decreased on the load of the cover body, the cover body deformation decreases. At the same time, due to the pressure and the deformation of the cover body is reduced, the stress of the cover body is reduced, and the service life of the cover body material is improved.

\section{Summary}

Based on the gas mask cover one-way FSI analysis, in the human body after wearing a mask in the suction process, the transient pressure distribution generated by the inspiratory airflow uneven load charge to a gas mask cover structure. The results of simulation and analysis of gas mask cover are 
more accurate and close to the actual situation. At the same time, the results of this paper can provide a theoretical basis for the further optimization of gas mask.

(1)By appropriately changing the size of the air inlet on the gas inlet and the water inlet of the water mask on the gas mask body, the force generated by the gas is reduced during the suction process, and the deformation is reduced.

(2)By changing the number of reinforcement at the bottom of the gas mask, the strength of the bottom of the cover body is increased, and the bottom deformation of the cover body is reduced.

\section{Reference}

[1] Y.T. Wang,X.Y. Li,L. Li, H.G. Li and X.L Huangfu: China Individual Protection Equipment,(2012) No.4,p.10.(In Chinese)

[2] Y.Ding,and Q.Wang.ICEM CFD ANSYS from Entry to the Master (Tsinghua University Publications, China 2013)p.7

[3] H.F. Zhang:Study on Aerodynamic Characteristics of Automobile Based on Turbulence Model (MS.,Hunan University,China 2011),p.27

[4] F.J. Wang.Principle and Application of Computational Fluid Dynamics Analysis -CFD Software (Tsinghua University Publications, China 2004)p.4

[5] J.Zhang:Fluid Mechanics Analysis and Modeling of Respiratory Protecttive Mask (MS.,Wuhan University of Technology,China 2011),p.6

[6] F. Wang and X.F. Wang: Journal of PLA Institute of Physical Education, Vol.22 (2003) No.4, p.76.

[7] L.H. Xie and X.Y. Zhao.CFX ANSYS Fluid Analysis and Simulation (Electronic Industry Publishing House, China 2013)p.105

[8] T.Zhang,X.J.Zhu and F.Peng: Journal of Naval University of Engineering,Vol. 25 (2013) No.6,p.104

[9] Z.M. Zhou:Study on the Structure and Properties of the Complex Modified Nylon 1010(MS.,Guizhou University,China 2013),p.15

[10]X.Y. Liu,H.F.Chang,X.G. Cai and W.Gao: Elastomer,Vol, 13 (2003) No.1,p.23 